\title{
Importance of In Vitro Microenvironment on Differentiation and Translational Applications of Dental Pulp Stem Cells
}

Li Q*

Cell Therapy Laboratory, The First Hospital of Hebei Medical University, Shijiazhuang, Hebei, China

*Corresponding author: Quanhai Li, Cell Therapy Laboratory, The First Hospital of Hebei Medical University, Shijiazhuang, Hebei 050031, China

Received: May 27, 2021; Accepted: J une 19, 2021; Published: J une 26, 2021

\begin{abstract}
Dental Pulp Stem Cells (DPSCs) are adult stem cells found in dental pulp tissue, and possess the capacity for self-renewal and the potential for multipotent differentiation. DPSCs depend on regulating in vitro microenvironment, and can readily differentiate into osteoblasts, odontoblasts, neurocytes, adipocytes, chondrocytes, myocytes, fibrocytes and many others. In addition, DPSCs play a crucial role in tooth regeneration, bone and nerve repair in current studies of regenerative medicine and tissue engineering. Among them, the influences of stem cell microenvironment or niche on the biological activity of DPSCs are critical and hamper its progress. Herein, we review the influence of culture condition, tissue source, growth factor requirements, and cellular organizational scaffolds and how these features influence the biological characteristics and translational research of DPSCs.
\end{abstract} Vitro

Keywords: Dental pulp stem cells; Differentiation; Microenvironment; In

of DPSCs

\section{Introduction}

Dental pulp is an integral part of tooth tissue and comprises connective tissue, mesenchymal cells, nerve fibers, blood vessels, lymph, and other biological components. Dental Pulp Stem Cells (DPSCs), which are also known as post-natal dental pulp stem cells, are adult stem cells located in dental pulp tissues. DPSCs were first isolated from the pulp tissues of the third molar impacted teeth by Gronthos et al. [1]. DPSCs are characterized as high clonogenic cells and proliferative capacity that can generate densely calcified colonies and occasional nodules. Furthermore, DPSCs are comprised predominantly of stem cells that are separated from dental pulp tissues of permanent teeth and from human exfoliated deciduous teeth (SHED). In 2003, Miura and colleges [2] isolated stem cells from the pulp tissue of SHED and found that SHED represented a population of post-natal stem cells that were capable of extensive proliferation and multipotent differentiation. Under different in vitro microenvironmental conditions, DPSCs can differentiate into many cell types like odontoblasts $[3,4]$, neural cells $[5,6]$, osteoblasts $[7,8]$, chondrocytes $[9,10]$, adipocytes [11], hepatic cells $[12,13]$, myoblasts [14], fibroblasts [15] and islet-like cells [16,17]. The cell culture environment also poses significant challenges in stem cell research, especially when there is a need to guarantee the undifferentiated state and directional differentiation of the cultured stem cells. Therefore, cell culture conditions are mostly defining by the requirements of the desired target cells. The specific cultural microenvironment such as inflammatory, acidic, and high glucose could affect differentiation of DPSCs through complex mechanisms. Moreover, the surrounding microenvironment also regulates DPSCs differentiation and proliferation. In addition, cell culture method applied, the tissue source of stem cells, supplementation with defined growth factors and cellular scaffolds could differentially influence the differentiation

\section{Influence of Cullture Condition on DPSCs Differentiation}

DPSCs had the ability to differentiate into various cell types under specific culture conditions. Several studied have revealed great potential for DPSCs in the repair and regeneration of various tissues, including bone [18], teeth [19], and muscles [20]. Kanafi and colleges [21] applied the alginate acid sodium salt microspheres to fix DPSCs three weeks, they founded that more than $80 \%$ of immobilized DPSCs remained viable and proliferation compared with the control group. On day 21, osteocalcin and calcium deposition were found in the microspheres and the expression of the osteogenic genes Alkaline Phosphatase (ALP), runt homology domain transcript factor (Runx-2) and Osteocalcin (OCN) were all up-regulated by Reverse Transcriptase Polymerase Chain Reaction Assay (RT-PCR). They conducted that DPSCs immobilized in alginate hydrogels could enhance osteogenic potential differentiation which was merit to bone tissue regeneration. Umemura and colleges [22] applied hyaluronic acid sodium salt to fix DPSCs, they founded that hyaluronan induced odontoblastic differentiation and the expression of the odontoblastic differentiation markers Dentin Matrix Protein 1 (DMP-1) and Dentin Sialophosphoprotein (DSPP) were up-regulated using western blot analysis. Nakayama and colleges [19] isolated DPSCs from mature teeth and mobilized them by Granulocyte ColonyStimulating Factor (G-CSF), they founded that mobilized DPSCs had higher differentiation, migration and regeneration potential ability compared to DPSCs. The differentiation procedure was in part mediated by the function of osteoblasts to secrete cytokines or soluble proteins. Kanafi and colleges [23] investigated the influence of hypoxia, high glucose and low serum concentration on the differentiation potential of human SHED. They founded that SHED 
retained their phenotypic expression and differentiation potential in different cultural conditions described above. They demonstrated that SHED possessed strong capacity of proliferation and differentiation even in harsh culture conditions.

\section{Influence of Tissue Source on DPSCs Differentiation}

Obviously, fresh teeth or healthy teeth alone will not satisfy clinical needs in the future application of DPSCs in cell-based therapy. Some studies have shown that inflamed pulp tissue of human functional deciduous teeth (iSHFD), carious deciduous teeth and cryopreserved teeth have similar differentiation capabilities to fresh tissue. Kim and colleges [24] reported that they successfully isolated Mesenchymal Stem Cells (MSCs) from iSHFD and the characteristics of stem cells were similar to that of healthy DPSCs. In addition, Fibroblast Growth Factor 2 (FGF-2) promoted the proliferation and migration of stem cells derived from inflamed dental pulp. It means that iSHFD could be used as seed cells in tissue repair engineering. A study by Werle and colleges [25] has shown that the success rate of isolation were $70 \%$ and $80 \%$ from stem cell from the pulp of carious deciduous teeth (SCCD) and sound deciduous teeth (SHED-stem cells from human exfoliated deciduous teeth) separately and SCCD had the similar pattern of proliferation and differentiation ability as those obtained from sound deciduous teeth. They concluded that SCCD could represent a feasible source of stem cells. Malekfar and colleges [26] successful isolated human DPSCs from fresh and cryopreserved pulp tissues with irreversible pulpitis respectively. They found that there was no significant difference in osteogenic and adipogenic differentiation potential of DPSCs between the fresh and frozen dental pulp tissues derived stem cells. They concluded that cryopreservation did not lose their normal characteristics and differentiation potential.

\section{Influence of Growth Factors on DPSCs Differentiation}

\section{Influence of growth factors on DPSCs osteogenic differentiation}

Several studies have shown that some kinds of growth factors such as Enamel Matrix Derivative (EMD), Vascular Endothelial Growth Factor (VEGF), insulin et al. can have positive effects on DPSCs osteogenic differentiation. Ajlan and colleges [27] added EMD, Mineral Trioxide Aggregate (MTA) and Platelet-Derived Growth Factor (PDGF) into DPSCs, respectively. It was shown that EMD significantly increased the expression of Alkaline Phosphatase (ALP) and mineralization compared to the other groups $(\mathrm{P}<0.05)$ by computer analysis of ALP-stained chambers and spectrophotometric analysis of alizarin red S-strained mineralized nodule. It suggested that EMD possessed the capacity to promote osteogenic differentiation of human DPSCs. VEGF is another kind of growth factor the can influence the proliferation and osteogenic differentiation of DPSCs which has been demonstrated by D' Alimonte and colleges [28]. They incubated DPSCs in osteogenic culture medium for three weeks and found that the expression of osteogenic markers (Runt- Related Transcription Factor 2 (RUNX-2), and ALP) had been increased, as did the expression of extracellular calcareous deposition. The influence of Endothelial Cells (ECs) on the osteogenic differentiation of DPSCs had been investigated by several studies. Dissanayaka et al. [29] co-incubated different ratios of Endothelial Cells (ECs) with DPSCs, and found that direct co-culture of DPSCs and ECs enhances the in vitro differentiation toward osteo-/odontogenic and angiogenic phenotypes. When the ratio was set at $1: 1$, the increase in ALP activity was the most significant. When the ratios were set at 1:1 and 1:5, the mineralization was stronger than was observed at other ratios $(\mathrm{P}<0.01)$. It suggested that ECs is able to enhance the osteogenic differentiation of human DPSCs. It is wellknown that insulin is a powerful and important hormone involved in the proliferation and differentiation of osteogenic. Lauritano and colleges [30] treated DPSCs in insulin $(100 \mathrm{ng} / \mu \mathrm{g})$ for $24 \mathrm{~h}$ and $48 \mathrm{~h}$, and compared the gene expression profiles in DPSCs in the insulintreated group and the non-treated group. It had founded that after 24 hours treatment, the expression of bone morphogenetic protein family members Bone Morphogenetic Proteins 3 (BMP3), BMP4, and their receptor BMPR1A were increased, and after 48 hours treatment, BMP1 was over-expressed. However, 24 hours after therapy, the expression of BMP7 and Transforming Growth Factor Beta Receptor (TGFBR) 2 had decreased. They conducted that insulin had positive effect on osteogenic differentiation of DPSCs. Several studied have reported that Valproic Acid (VPA) is a Histone Deacetylase (HDAC) inhibitor, which promotes osteoblastic differentiation of HPSCs. Paino and colleges [7] found that low concentration of VPA increased the expression of osteopontin and bone sialoprotein, and further significantly improved bone mineralization. However, the osteocalcin level decreased, which was significant related to the presence of an HDAC2 inhibitor. Accordingly, they concluded that VPA did not induce terminal differentiation of osteogenic, but just stimulated the generation of less mature cells, and specific suppression of the individual HDAC by RNA interference could only a single aspect of osteogenic differentiation. It had proved that Tumor Necrosis Factor- $\alpha$ (TNF- $\alpha$ ), a major pro-inflammatory cytokine, with low concentration could promote osteogenic differentiation of DPSCs [31]. While in higher concentration which could decreased the capability of osteogenic differentiation [32], which may be affected by nuclear factor kappa-light-chain- enhancer of activated B cell (NF$\mathrm{kB}) / \mathrm{p} 38$ mitogen-activated protein kinases (p-38 MAPK)/Wnt/ $\beta$ caternin signal pathway $[33,34]$. The exact underlying molecular mechanisms are need to further study.

\section{Influence of growth factors on DPSCs odontoblastic differentiation}

It previously reported that under the induction of a certain factor, DPSCs possessed the ability to migrate, and stimulated the proliferation and differentiation of DPSCs to form dentin, which is an important biological pathway in the regeneration of teeth and dental pulp after dental pulp-dentin damage. The Transforming Growth Factor (TGF) $-\beta$ family is composed of polypeptide growth factors, including BMPs, TGF- $\beta$, activin and growth differentiation factor. TGF- $\beta$ plays important roles in embryonic development, extracellular matrix formation, bone remodeling and formation. Besides, it also influences cell proliferation and differentiation, and TGF- $\beta$ is closely related to odontoblast differentiation and dentin matrix formation. Wiesen et al. [15] evaluated the expression of periostin with different concentration of TGF- $\beta 1$ by RT-PCR, western blot and Enzyme-Linked Immunosorbent Assay (ELISA) in the activity of DPSCs, Dental Pulp Fibroblasts (DPFs), and rat odontoblast-like cells (MDPC-23). After 48 hours of TGF- $\beta 1$ treatment, the expression of periostin mRNA increased in the DPSCs group and decreased in 
the DPFs group. These observations indicated that TGF- $\beta 1$ could promote DPSCs odontoblastic differentiation. Semaphorin was identified by Kolodkin et al. in 1992, and is a type of secretory or transmembrane family member, which contains the Sema structural domain [35]. Sema3A expression (originally referred to as Collapsin), belongs to a subset of the semaphorin family, and was first discovered by Luo et al. [36] in 1993. In 1999, the Semaphorin Nomenclature Committee divided this family into eight sub-types, and twenty members were identified in the mouse and human [37]. Yoshida and colleges [38] applied immunohistochemistry to detect the expression of Semaphorin 3A (Sema 3A) and its receptor (neuropilin 1) in rat DPSCs model. Sema3A induced DPSCs migration, proliferation and odontoblastic differentiation and increased nuclear accumulation of $\beta$-catenin and expression of the FARP2 gene (FERM, RhoGEF, and pleckstrin domain protein 2), and improved Racl activity. They conducted that Sema3A played a crucial role in tooth regeneration and the possible molecular mechanism might be the Wnt $/ \beta$-catenin signaling pathway. The differentiation inhibitor which also referred to as the inhibitor of differentiation/DNA binding (Id) belongs to one of the Helix-Loop-Helix (HLH) transcription factor family members. There are four Id molecules in mammalian cells (i.e., Id1-Id4) [39]. Maciejewska and colleges [40] reported that Id1 over-expression could influence the differentiation of human DPSCs. Over-expression of Id1 increased the expression of the Dentin Sialophosphoprotein (DSP) and the gene expression of Dentin Matrix Protein 1 (DMP1), and further increased odontoblastic differentiation, while Id1 silencing showing opposite effect to DPSC differentiation. The results suggest that as a vital modulator Id1 plays important role during DPSC differentiation. A study by Liu and colleges has shown the ECs have influence on the odontogenic differentiation of DPSCs [41]. Liu and colleges co-cultured Human Umbilical Vein Endothelial Cell (HUVEC) to DPSCs and the expression of DSP and DMP-1were detected by RT-PCR, Alizarin Red S staining, immunofluorescence and western blotting analysis at day 4, 7, 14 and 21. They founded that HUBEC group exhibited higher odontoblastic differentiation compared to the controls groups and the highest expression levels of DSP and DMP-1 were at day 7. However, recently a kind of growth factor named Casein Kinase 2 Interacting Protein 1 (CKIP1) was reported that can suppress odontoblastic differentiation of DPSCs by Song and colleges [42]. They co-cultured DPSCs and CKIP-1 in odontoblastic differentiation medium for 21 days and the expression of CKIP-1, BMP2, Neuropilin-1 (NRP1) were detected by RT-PCR, western blot, ALP straining, alizarin red S staining and immunoprecipitation. The CKIP-1 expression significantly decreased along with cell odeontoblastic differentiation, while CKIP-1 over expression had a negative effect on odentoblastic differentiation of DPSCs. It provides a new perception on odontoblastic differentiation of DPSCS and may be of great significance to the repair of dental caries.

\section{Influence of growth factors on DPSCs neurogenic differentiation}

DPSCs derived from the ectoderm, and their capacity of differentiating into the neural lineage is higher than that of cells derived from other blastodermal development sites [43]. Several studies have shown that Basic Fibroblast Growth Factor (bFGF) had positive effects on neurogenic differentiation of DPSCs. A study by Kanafi and colleges [44] was shown that DPSCs in their naïve state spontaneously expressed early and mature neuronal markers like nestin, musashi12, $\beta$ tubulin III and on exposure to bFGF, DPSCs expressed high level of dopaminergic neuron-specific transcription factors Nuclear Receptor related protein 1 (Nurr1), Engrailed 1 (En1) and paired-like homeodomain transcription factor 3 (Pitx3) as revealed by RT-PCR. Another study by Osathanon and colleges [45] confirmed that bFGF could promote proliferation and neurogenic differentiation of DPSCs. The other growth factor can influence neurogenic potential differentiation of DPSCs is p75. Pan and colleges [46] investigated DPSCs from eight donors, and found that p75 neurotrophin receptors (p75NTR) were expressed at low levels $(<10 \%)$ in DPSCs and p75 $(+)$ DPSC exhibited higher expression levels of SOX1 (a neural precursor cell marker), SOX2 (a cell pluripotency marker) and nestin (a neuronal stem cell marker) as compared to p75(-) DPSCs. It suggested that p75 (+) human DPSCs possessed greater neurocytic differentiation potential.

\section{Influence of growth factors on DPSC myogenic differentiation}

MicroRNA can be considered as a type of endogenous, single stranded non-coding RNA with an average length of approximately $18 \sim 24 \mathrm{nt}$. It plays vital roles in gene regulation and is involved in many biological processes, including cell proliferation, differentiation, aging and apoptosis [47]. Li and colleges [14] applied miR-143 and miR-135 inhibitors to observe their influence on DPSC myogenic differentiation by RT-PCR and western blotting. They founded that the expression of miR-135 and miR-143 markedly decreased in DPSCs and part of the DPSCs treated with miR-135 or miR-143 inhibitors displayed apparent myocytic properties, and formed myotubes. In addition, in complementary studies, miR-135 and miR143 inhibitors were transfected into DPSCs, following which half the DPSC population formed myotubes. It indicated that miR-135 and miR-143 inhibitors could induce myogenic differentiation of DPSCs.

\section{Influence of Different Scaffolds on DPSC Differentiation}

Different scaffolds provide different microenvironmental niches for DPSCs, which can provoke differential influences on DPSC differentiation, as described in the examples provided below.

\section{Scaffold influencing osteogenic differentiation}

Annibali et al. [48] seeded DPSCs on two molecular scaffolds, Granular Deproteinized Bovine Bone (GDPB) and Beta-Tricalcium Phosphate ( $\beta$-TCP). The repair and regenerative functions of both molecular scaffolds on the damaged cranium were comparing in a rat model. After 2, 4, 8 and 12-week post-implant, Bone Mineral Density (BMD) and the Standard Uptake Value (SUV) of tracer incorporation were measured, respectively. The repair of bone formation mediated by the interplay of GDPB in the damaged cranium was better than $\beta$-TCP. It suggests that GDPB scaffolds could improve bone regeneration of DPSCs. Niu et al. [49] investigate the effects of Intrafibrillar-Silicified Collagen Scaffolds (ISCS) on the osteogenic differentiation of human DPSCs by RT-PCR, western blotting, ALP staining and alizarin red S assay. They co-incubated ISCS with DPSCs, and founded that ISCS could promote DPSCs proliferation and significantly increased expression of osteogenesis-related genes and proteins during DPSCs osteoblastic differentiation. It is more important that there is no adverse effect on human DPSCs. Lobo et 
al. [50] co-incubated Biphasic Calcium Phosphate (BCP) biological ceramics scaffolds with DPSCs. The number of surviving DPSCs in the co-culture group was higher than that in the control group. BCPs with a small pore size promoted cell proliferation, and those with a larger pore size increased expression of osteogenic-related genes and proteins during osteogenic differentiation, which showed that BCPs might possess a bone inductive potential. Farea et al. [51] proved that the combination of a chitosan scaffold and TGF- $\beta 1$ could increase proliferation and osteoblastic differentiation of SHED. The combination of the chitosan scaffold and TGF- $\beta 1$ improved bone tissue regeneration with SHED in vivo. It has shown that Hydroxyapatite (HAp) can support alginate as inorganic reinforcement and osteoconductive component of alginate/HAp composite scaffolds. A study by Sancilio and colleges [52] co-incubated Alg/HAp scaffolds to human DPSCs and they founded that when growing onto Alg/HAp scaffolds DPSCs expressed osteogenic differentiation markers and promoted calcium depositing and biomineralization. In summary, all kinds of scaffold above mentioned have positive effects on the proliferation and osteoblastic differentiation of DPSCs to a certain extent, and future research in animal model and clinical experience should confirmed to guarantee the safety and effectiveness.

\section{Scaffold influencing odontogenic differentiation}

Conde et al. [53] used WST-1 assay and RT-PCR to detect the influence of poly -L-lactic acid scaffolds with different pore sizes (i.e., $150-250 \mu \mathrm{m}$ or $251-450 \mu \mathrm{m}$ ) on the proliferation and differentiation of DPSCs. They found that after 21 days, DPSCs showed odontogenic gene expression of DMP-1, DSPP, and Matrix Extracellular Phosphoglycoprotein (MEPE). It concluded that the poly-L-lactic acid scaffold promoted cell proliferation and odontoblastic differentiation. It is well known that the differentiation and regeneration potential of stem cell including DPSCs depends on their environment. A study by Soares and colleges [54] seeded lipopolysaccharide (LPS) or simvastatin and/or nanofibrous poly-l-lactic acid to human DPSCs, they founded that in vitro studies using simvastatin and nanofibrous poly-l-lactic acid scaffolds significantly repress the expression of proinflammatory mediators and reverted the negative effects of LPS on expression of odontoblastic markers in vivo in mouse model. It may provide a new strategy for DPSCS to treat inflammation and dental tissue regeneration. Cavalcanti et al. [55] co-incubated DPSCs with $0.05-0.25 \%$ Puramatrix ${ }^{\mathrm{TM}}$ DPSCs survived and proliferated well in Puramatrix $^{\text {in }}$ at least three weeks and kept healthy morphology, and part of the cytoplasm lengthened. After 21 days, DMP-1 and DSPP were expressing in DPSCs with Puramatrix ${ }^{\mathrm{TM}}$. The peptide hydrogel scaffold could be used as a form of gene therapy and provided support in tooth tissue regeneration.

\section{Scaffold influencing neurogenic differentiation}

Chitosan is a natural macromolecule and a kind of biodegradable material that has been widely used in clinics. In terms of having similar structure and composition to Glycosaminoglycans (GAGs) chitosan elicits minimal immune response when implanted in human body. Therefore, chitosan is able to use as an ideal biomedical scaffold material. Feng et al. [56] seeded DPSCs on a three-dimensional (3-D) porous chitosan scaffold, and incubated them with neural induction medium in vitro and they founded that the neurogenic differentiation markers were significantly increased. Zhang et al. [57] incubated DPSCs with chitosan scaffold in neural induction medium for 14 days, and they founded that compared the control rats, the DPSCs/ chitosan group showed the higher cell neural differentiation and the expression of Brain-Derived Neurotrophic Factor (BDNF), Glial Cell Line-Derived Neurotrophic Factor (GDNF), b-Nerve Growth Factor (b-NGF) and Neurotrophic-3 (NT-3) were significantly upregulated by western blotting assay and RT-PCR. Transplantation of DPSCs with chitosan scaffolds into the injured rat model resulted in the significant recovery of hind limb function. It suggested that the scaffold provided a conducive and favorable microenvironment for the survival and neuronal differentiation of DPSCs.

\section{Application of DPSCs in Tissue Engineering and Regenerative Medicine}

DPSCs have acceptable efficacy in the repair and regeneration of teeth, bone and nerve tissues, and can achieve significant progress in tissue biology and regenerative medicine, possessing the potential for a wide array of applications.

\section{Animal experiment}

Several animal experiments have approved that DPSCs had excellent efficacy in neural repair. Ullah et al. [58] transplanted a total of $1 \times 10^{6}$ human DPSCs with fibrin glue scaffold and collagen tubulization into the sciatic nerve resection ( $5 \mathrm{~mm}$ gap) of rat models. After 12 weeks, human DPSCs groups showed notably increased behavioral activities and higher muscle contraction forces compared with control group and regenerated nerve specimens, specific markers for angiogenesis, axonal fiber, and myelin sheath increased by immunohistochemical analysis in DPSCs transplantation groups. They demonstrated that human DPSCs could be an excellent stem cell source for nerve regeneration. Zhang et al. [59] evaluated the therapeutic potency of DPSCs and BDNF in focal cerebral ischemia using rat models. They transplanted DPSCs and injected BDNF into the rats via the tail vein. Proliferation of intravenously transplanted DPSCs and the expression of neural differentiation markers such as nestin, Doublecortin (DCX) and Neuronal Specific Filament (NF$\mathrm{H}$ ) were founded in the peripheral ischemic regions of the Middle Cerebral Artery Occlusion (MCAO) models. They conducted that treatment with DPSCs combined with BDNF promoted the recovery of neurological function after stroke which suggesting a novel therapeutic strategy against cerebral ischemia. A study by Pisciotta et al. [60] has shown that human DPSCs had potential efficacy in skeletal muscle repair in mice. They co-cultured human DPSCs with myogenic cell line (C2C12 cells) and preliminary demethylation treatment with 5-Aza-2'-deoxycytidine (5-Aza), respectively in vitro for 2 weeks. Then the cells were injected into the dystrophic gastrocnemius muscles of $\mathrm{mdx} / \mathrm{SCID}$ mice. After 1, 2, and 4 weeks, the gastrocnemius muscles were taken for immunofluorescence and histological analyses. They founded that the populations of cells engrafted within the host muscle of mdx/SCID mice improved, angiogenesis promoted and fibrosis reduced through a paracrine effect, which eventually led to an improvement of the histopathology of the dystrophic muscle. During that last decade, a number of experiments showed the successful application of DPSCs in achieving large bone volume regeneration. Perczel-Kovach et al. [61] implanted DPSCs on osseointegration in rat tail vertebrae and they founded that undifferentiated DPSCs inserted between the implant and the bone slow down the osseointegration process and 
Table 1: Influence of in vitro microenvironmental niches on DPSCs Differentiation.

\begin{tabular}{|c|c|c|c|c|}
\hline Name of Niche & Factor in Detail & Differentiated Direction & Involved Molecules or Pathway & Reference \\
\hline \multirow{2}{*}{ Cultural Condition } & $\begin{array}{l}\text { Alginate Microspheres } \\
\text { Fixture }\end{array}$ & Osteogeni Differentiation & ALP, Runx-2 and OCN & {$[21]$} \\
\hline & $\begin{array}{l}\text { Hyaluronic Acid Sodium } \\
\text { Salt }\end{array}$ & Odontogenic Differentiation & DMP-1, DSPP & [22] \\
\hline \multirow{3}{*}{ Issue Source } & Inflamed Dental Pulp & Odontogenic Differentiation & FGF-2 & [24] \\
\hline & Carious Deciduous Dental & $\begin{array}{c}\text { Osteogenic, Chondrogenic and Adipogenic } \\
\text { Differentiation }\end{array}$ & $\mathrm{CD}^{29}+\mathrm{CD}^{+} 3^{+} / \mathrm{CD} 90^{+} / \mathrm{CD} 14 / \mathrm{CD} 34 / \mathrm{CD} 45^{-}$ & {$[25]$} \\
\hline & Crypreserved Dental Pulp & Osteogenic and Adipogenic Differentiation & $\begin{array}{l}\mathrm{CD} 166^{+} / \mathrm{CD} 105^{+} / \mathrm{CD} 90^{+} / \mathrm{CD} 73^{+} / \mathrm{CD} 45 \% \\
\mathrm{CD} 34 / \mathrm{CD} 19\end{array}$ & {$[26]$} \\
\hline \multirow{13}{*}{$\begin{array}{l}\text { Growth Factor } \\
\text { Requirements }\end{array}$} & EDM & Osteogenic Differentiation & $\mathrm{CD}^{2} 3^{+} / \mathrm{CD} 90^{+} / \mathrm{CD} 105^{+} / \mathrm{CD} 45 / \mathrm{CD} 34^{\prime} / \mathrm{CD} 14^{-}$ & [27] \\
\hline & VEGF & Osteogenic Differentiation & RUNX-2, and ALP & [28] \\
\hline & \multirow{2}{*}{ ECs } & Osteogenic Differentiation & ALP Activity & [29] \\
\hline & & Odentogenic Differentiation & DSP, DMP-1 & [36] \\
\hline & Insulin & Osteogenetic Differentiation & BMP3 ,BMP4 and BMPR1A & {$[30]$} \\
\hline & VPA & Osteogenic Differentiation & Osteopontin and Bone Sialoprotein & [31] \\
\hline & TNF-a & Osteogenic Differentiation & NF-KB/p38, Wnt/ß-catenin Signaling & {$[32]$} \\
\hline & TGF-b1 & Odontogenic Differentiation & Periostin & [33] \\
\hline & Sema A & Odontogenic Differentiation & FARP2 gene & [34] \\
\hline & Id1 & Odontogenic Differentiation & DSF, DMP-1 & [35] \\
\hline & CKIP-1 & Odontogenic Differentiation & BMP2 NRP1 & {$[37]$} \\
\hline & bFGF & Neurocytic Differentiation & Nestin, Musashi12, $\beta$ tubulin III & {$[44,45]$} \\
\hline & $\begin{array}{l}\text { miR-143, miR-135 } \\
\text { inhibitors }\end{array}$ & Myogenic Differentiation & Formed Myotube & [14] \\
\hline \multirow{9}{*}{ Organizational Scaffolds } & GDPB & Osteogenic Differentiation & BMD, SUV & [48] \\
\hline & ISCS & Osteogenic Differentiation & $\mathrm{OCN}$ & [49] \\
\hline & BCP Biological Scaffold & Osteogenic Differentiation & BSP, OPN & [50] \\
\hline & Chitosan Scaffold & Osteogenic Differentiation & ALP, COL1, BSP, OCN & {$[51]$} \\
\hline & Alg/Hap Scaffold & Osteogenic Differentiation & BMP2, RUNX2, SP7 & {$[52]$} \\
\hline & poly-L-lactic Acid Scaffolds & Odontoblastic Differentiation & DMP-1, DSPP and MEPE & {$[53,54]$} \\
\hline & Puramatrix ${ }^{\mathrm{TM}}$ & Odontoblastic Differentiation & MP-1 and DSPP & [55] \\
\hline & $\begin{array}{l}\text { 3-D Porous Chitosan } \\
\text { Scaffold }\end{array}$ & Neuroblastic Differentiation & Nestin, MSP2, GFA & {$[56]$} \\
\hline & Chitosan Scaffold & Neuroblastic Differentiation & BDNF, GDNF, b-NGF and NT-3 & [57] \\
\hline
\end{tabular}

DPSCs: Dental Pulp Stem Cells; EDM: Enamel Matrix Derivative; VEGF: Vascular Endothelial Growth Factor; ECs: Endothelial Cells; VPA: Valproic Acid; TNF-a: Tumor Necrosis Factor-a; TGF: Transforming Growth Factor; Sema 3A: Semaphorin 3A; Id: Inhibitor of Differentiation/DNA Binding; CKIP1: Casine Kanase 2 Interacting Protein 1; bFGF: Basic Fibroblast Growth Factor; GDPB: Granular Deproteinized Bovine Bone; ISCS: Intrafibrillar-Silicified Collagen Scaffolds; Alg/Hap: Alginate/Hydroxyapatite.

pre-differentiated DPSCs did not have the similar adverse effect. They suggested that the success of DPSCs application to promote implant osseointegration was highly dependent on the applied conditions, particularly on the parallel application of scaffolds and osteogenic components. Accordingly, efficient and safety scaffolds should be further studied in the future. It had shown DPSCs could be used for stem cell therapy in diabetes. Kanafi et al. [17] found that both SHED and DPSCs could generate Islet-Like Cell Clusters (ICCs), but SHED was superior to DPSCs. Streptozotocin (STZ)-induced diabetic mice alone and mice transplanted with empty macro-capsules both exhibited hyperglycemia throughout the experiment. Whereas mice transplanted with macro-capsules containing ICCs restored to normoglycemia within 3 to 4 weeks, which persisted for more than 60 days. In summary, we think that DPSCs play an important role in the repair and regeneration of tissues including nerves, bones, and muscles, further research should studied.

\section{Clinical applications}

There are few clinical applications of DPSCs in practice and just a small number of bone repair treatments trial. Manimaran et al. [62] applied autologous DPSCs and Stromal Vascular Fraction (SVF) with the intent of evaluating bone repair therapy on the post-operative bone defect of a 14-year-old male patient presenting with mandibular ameloblastoma. Ten month post-surgery, bone formation was observed by CT scanning, and no tumor relapse was reported during the follow-up observation. Li et al. [63] located autologous DPSCs onto the $\beta$-tricalcium phosphate scaffolds and engrafted them into the periodontal defect area in the root furcation of defect teeth. Nine-month post-surgery, the effect of regeneration of new bones to repair periodontal defects been verified by clinical assessment and radiological study. Ferrarotti et al. [64] random divided 29 chronic periodontitis patients who presented one deep intrabony defects and tooth had to be removed into DPSCs treatment 
group ( $\mathrm{n}=15)$ and the control group ( $\mathrm{n}=14)$. After 1-year treatment, they founded that test sites significantly improved clinical parameters regeneration in autologous DPSCs group compared to control patients. All of the above are autografts, the following case is an example of allogeneic transplantation. Hernández-Monjaraz et al. [65] applied SHED of a 7-year-old donor with scaffold of lyophilized collagen-polyvinylpyrrolidone sponger to a 61-year-old patient with periodontal disease. Six months later, the effect of regeneration of periodontal defects verified. All these clinical trials of the DPSCs are exciting and meaningful which laid a solid foundation on the further applications.

In summary, DPSCs have acceptable efficacy on repair and regeneration of tooth tissue, bone tissue and nerve tissue, and achieve significant progress in tissue biology and regenerative medicine - thus displaying prospects for their wide clinical application. The cell source, culture method and other in vitro microenvironmental niches (Table 1) may all contribute to differentiation characteristics. Currently, research studies on the mechanism of in vitro microenvironment on DPSC differentiation remain incomplete. With continuous development of research advances in the field, DPSCs will play a more significant role in tissue engineering.

\section{References}

1. Gronthos S, Mankani M, Brahim J, Robey PG, Shi S. Postnatal human Denta Pulp Stem Cells (DPSCs) in vitro and in vivo. Proc Natl Acad Sci USA. 2000; 97: 13625-13630.

2. Miura M, Gronthos S, Zhao M, Lu B, Fisher LW, Robey PG, et al. SHED: Stem cells from human exfoliated deciduous teeth. Proc Natl Acad Sci USA. 2003; 100: 5807-5812.

3. Zhao FL, Liu XY, Wang XB. The Role of MicroRNA-143-5p in the Differentiation of Dental Pulp Stem Cells into Odontoblasts by Targeting Runx2 via the OPG/ RANKL Signal Pathway. J Cell Biochem. 2018; 119: 536-546.

4. Maciejewska I, Sakowicz-Burkiewicz M, Krzeminska M, Pawelczyk T. Overexpression of ID1 reverses the repression of human dental pulp stem cells differentiation induced by TWIST1 silencing. Acta Biochim Pol. 2017; 64: 615-619.

5. Zhang J, Lian M, Cao P, Bao G, Xu G, Sun Y, et al. Effects of Nerve Growth Factor and Basic Fibroblast Growth Factor Promote Human Dental Pulp Stem Cells to Neural Differentiation. Neurochem Res. 2017; 42: 1015-1025.

6. Bojnordi MN, Haratizadeh S, Darabi S, Hamidabadi HG. Neural derivation of human dental pulp stem cells via neurosphere technique. Bratisl Lek Listy. 2018; 119: 550-553.

7. Paino F, La Noce M, Tirino V, Naddeo P, Desiderio V, Pirozzi G, et al. Histone deacetylase inhibition with valproic acid downregulates osteocalcin gene expression in human dental pulp stem cells and osteoblasts: evidence for HDAC2 involvement. Stem Cells. 2014; 32: 279-289.

8. Tabatabaei FS, Torshabi M. In vitro proliferation and osteogenic differentiation of endometrial stem cells and dental pulp stem cells. Cell Tissue Bank. 2017; 18: $239-247$

9. Westin CB, Trinca RB, Zuliani C, Coimbra IB, Moraes AM. Differentiation of dental pulp stem cells into chondrocytes upon culture on porous chitosanxanthan scaffolds in the presence of kartogenin. Mater Sci Eng C Mater Bio Appl. 2017; 80: 594-602.

10. Carbone A, Valente M2, Annacontini L, Castellani S, Di Gioia S, Parisi D et al. Adipose-derived mesenchumal stromal (stem) cells differentiate to osteoblast and chondroblast lineages upon incubation with conditioned media from dental pulp stem cell-derived osteoblasts and auricle cartilage chondrocytes. J Bio Requl Homeost Agents. 2016; 30: 111-122.

11. Ikbale el-A, Goorha S, Reiter LT, Miranda-carboni GA. Effects of hTERT immortalization on osteogenic and adipogenic differentiation of dental pulp stem cells. Data Brief. 2016; 6: 696-699.

12. Chen YK, Huang AH, Chan AW, Lin LM. Human dental pulp stem cells derived from cryopreserved dental pulp tissues of vital extracted teeth with disease demonstrated hepatic-like differentiation. J Tissue Eng Regen Med. 2016; 10: 475-485.

13. Cho YA, Noh K, Jue SS, Lee Sy, Kim EC. Melatonin promotes hepatic differentiation of human dental pulp stems: clinical implications for the prevention of liver fibrosis. J Pineal Res. 2015; 58: 127-135.

14. Li D, Deng T, Li H, Li Y. MiR-143 and miR-135 inhibitors treatment induces skeletal myogenic differentiation of human adult dental pulp stem cells. Arch Oral Biol. 2015; 60: 1613-1617.

15. Wiesen RM, Padial-Molina M, Volk SL, McDonald N, Chieqo DJ, Botero T, et al. The expression of periostin in dental pulp cells. Archives of Oral Biology. 2015; 60: 760-767.

16. Govindasamy V, Ronald VS, Abdullah AN, Nathan KR, Ab Aziz ZA, Abdullah $M$, et al. Differentiation of dental pulp stem cells into islet-like aggregates. $J$ Dent Res. 2011; 90: 646-652.

17. Kanafi MM, Rajeshwari YB, Gupta S, Dadheech N, Nair PD, Gupta PK, et al. Transplantation of islet-like cell clusters derived from human dental pulp stem cells restores normoglycemia in diabetic mice. Cytotherapy. 2013; 15 : 1228-1236.

18. Asutay F, Polat S, Gül M, Subaşı C, Kahraman SA, Karaöz E. The effects of dental pulp stem cells on bone regeneraton in rat calvarial defect model: microcomputed tomography and histomorphometrics analysis. Arch Oral Biol. 2015; 60: 1729-1735.

19. Nakayama H, Iohara K, Hayashi Y, Okuwa Y, Kurita K, Nakashima M. Enhanced regeneration potential of mobilized dental pulp stem cells from immature teeth. Oral Dis. 2017; 23: 620-628.

20. Hata M, Omi M, Kobayashi Y, Nakamura N, Tosaki T, Miyabe M, et al. Tranplanation of cultured dental pulp stem cells into the skeletal muscles ameliorated diabetic polyneuropathy: therapeutic plausibility of freshly isolated and cryopreserved dental pulp stem cells. Stem Cell Res Ther. 2015; 6: 162

21. Kanafi MM, Ramesh A, Gupta PK Bhonde PP. Dental pulp stem cells immobilized in alginate microspheres for applications in bone tissue engineering. Int Endod J. 2014; 47: 687-697.

22. Umemura N, Ohkoshi E, Tajima M, Kikuchi H, Katayama T, Sakagami H. Hyaluronan induces odontoblastic differentiation of dental pulp stem cells via CD44. Stem Cell Res Ther. 2016; 7: 135.

23. Kanafi MM, Ramesh A, Gupta PK, Bhonde RR. Influence of hypoxia, high glucose, and low serum on the growth kinetics of mesenchymal stem cells from deciduous and permanent teeth. Cells Tissues Organs. 2013; 198: 198208.

24. Kim J, Park JC, Kim SH, Im GI, Kim BS, Lee JB, et al. Treatment of FGF-2 on stem cells from inflamed dental pulp tissue from human deciduous teeth. Oral Dis. 2014; 20: 191-204.

25. Werle SB, Lindemann D, Steffens D, Demarco FF, de Araujo FB, Pranke P, et al. Carious deciduous teeth are a potential source for dental pulp stem cells. Clin Oral Investig. 2016; 20: 75-81.

26. Malekfar A, Valli KS, Kanafi MM Bhonde RR. Isolation and Characterization of Human Dental Pulp Stem Cells from Cryopreserved Pulp Tissues Obtained from Teeth with Irreversible Pulpitis. J Endod. 2015; 42: 76-81.

27. Ajlan SA, Ashri NY, Aldahmash AM, Alnbaheen MS. Osteogenic differentiation of dental pulp stem cells under the influence of three different materials. BMC Oral Health. 2015; 15: 132.

28. D' Alimonte I, Nargi E, Mastrangelo F, Falco G, Lanuti P, Marchisio M, et al. Vascular endothelial growth factor enhances in vitro proliferation and osteogenic differentiation of human dental pulp stem cells. J Biol Regul Homeost Agents. 2011; 25: 57-69.

29. Dissanayaka WL, Zhan X, Zhang C, Hargreaves KM, Jin L, Tong EH. Coculture of dental pulp stem cells with endothelial cells enhances osteo- $/$ odontogenic and angiogenic potential in vitro. J Endod. 2012; 38: 454-463. 
30. Lauritano D, Avantaggiato A, Candotto V, Cura F, Gaudio RM, Martinelli M, et al. Insulin Activity On Dental Pulp Stem Cell Differentiation: An In Vitro Study. J Biol Regul Homeost Agents. 2015; 29: 48-53.

31. Feng X, Feng G, Xing J, Shen B, Li L, Tan W, et al. TNF-a triggers osteogenic differentiation of human dental pulp stem cells via the NF-KB signaling pathway. Cell Biol Int. 2013; 37: 1267-1275.

32. Huang H, Zhao N, Xu X, Xu Y, Li S, Zhang J, et al. Dose-specific effects of tumor necrosis factor alpha on osteogenic differentiation of mesenchymal stem cells. Cell Prolif. 2011; 44: 420-427.

33. Cui YM, Han XH, Lin YY, Lv WW, Wang YL. TNF- $\alpha$ was involved in calcium hydroxide-promoted osteogenic differentiation of human DPSCs through NFKB/p38MAPK/Wnt pathway. Pharmazie. 2017; 72: 329-333.

34. Feng G, Zheng K, Song D, Xu K, Huang D, Zhang Y, et al. SIRT1 was involved in TNF-a-promoted osteogenic differentiation of human DPSCs through Wnt/ $\beta$-catenin signal. In Vitro Cell Dev Biol Anim. 2016; 52: 1001-1011.

35. Kolodkin AL, Matthes DJ, O'Connor TP, Patel NH, Admon A, Bentley D, et al. Fasciclin IV: sequence, expression, and function during growth cone guidance in the grasshopper embryo. Neuron. 1992; 9: 831-845.

36. Luo Y, Raible D, Raper JA. Collapsin: A protein in brain that induces the collapse and paralysis of neuronal growth cones. Cell. 1993; 75: 217-227.

37. Goodman CS, Kolodkin AL, Luo Y, Püschel AW, Raper JA. Unified nomenclature for the semaphorins/collapsins. Semaphorin Nomenclature Committee. Cell. 1999; 97: 551-552.

38. Yoshida S, Wada N, Hasegawa D, Miyaji H, Mitarai H, Tomokiyo A, et al. Semaphorin 3A Induces Odontoblastic Phenotype in Dental Pulp Stem Cells. J Dent Res. 2016; 95: 1282-1290.

39. Sharma P, Chinaranagari S, Chaudhary J. Inhibitor of differentiation 4 (ID4) acts as an inhibitor of ID-1, -2 and -3 and promotes basic helix loop helix (bHLH) E47 DNA binding and transcriptional activity. Biochimie. 2015; 112: 139-150

40. Maciejewska I, Sakowiczburkiewicz M, Pawelczyk T. Id1 Expression Leve Determines the Differentiation of Human Dental Pulp Stem Cells. J Dent Res. 2014; 93: 576-581.

41. Liu M, Zhao L, Hu J, Wang L, Li N, Wu D, et al. Endothelial cells and endothelin 1 promote the odontogenic differentiation of dental pulp stem cells. Mol Med Rep. 2018; 18: 893-901.

42. Song Y, Wang C, Gu Z, Cao P, Huang D, Feng G, et al. CKIP-1 suppresses odontoblastic differentiation of dental pulp stem cells via BMP2 pathway and can interact with NRP1. Connect Tissue Res. 2019; 60: 155-164.

43. Kumar A, Kumar V, Rattan V, Jha V, Bhattacharyya S. Secretome Cues Modulate the Neurogenic Potential of Bone Marrow and Dental Stem Cells. Mol Neurobiol. 2017; 54: 4672-4682.

44. Kanafi M, Majumdar D, Bhonde R, Gupta P, Datta I. Midbrain cues dictate differentiation of human dental pulp stem cells towards functional dopaminergic neurons. J Cell Physiol. 2014; 10: 1369-1377.

45. Osathanon T, Nowwarote N, Pavasant P. Basic fibroblast growth factor inhibits mineralization but induces neuronal differentiation by human dental pulp stem cells through a FGFR and PLCy signaling pathway. J Cell Bioch. 2011; 112: 1807-1816

46. Pan W, Kremer KL, Kaidonis X, Ludlow VE, Rogers ML, Xie J, et al. Characterization of p75 neurotrophin receptor expression in human dental pulp stem cells. Int J Dev Neurosci. 2016; 53: 90-98.

47. Judson L, Babiarz J, Venere M, Blelloch R. Embryonic stem cell specific microRNAs promote induced pluripotency. Nature Biotechnology. 2009; 27: 459-461.

48. Annibali S, Bellavia D, Ottolenghi L, Cicconetti A, Cristalli MP, Quaranta $\mathrm{R}$, et al. Micro-CT and PET analysis of bone regeneration induced by biodegradable scaffolds as carriers for dental pulp stem cells in a rat model of calvarial "critical size" defect: Preliminary data. J Biomed Mater Res B App Biomater. 2014; 102: 815-825.
49. Niu LN, Sun JQ, Li QH, Jiao K, Shen LJ, Wu D, et al. Intrafibrillar-silicified collagen scaffolds enhance the osteogenic capacity of human dental pulp stem cells. J Dent. 2014; 42: 839-849.

50. Lobo SE, Glickman R, da Silva WN, Arinzeh TL, Kerkis I. Response of stem cells from different origins to biphasic calcium phosphate bioceramics. Cell Tissue Res. 2015; 361: 477-495.

51. Farea M, Husein A, Halim AS, Abdullah NA, Mokhtar KI, Lim CK, et al. Synergistic effects of chitosan scaffold and TGF $\beta 1$ on the proliferation and osteogenic differentiation of dental pulp stem cells derived from human exfoliated deciduous teeth. Arch Oral Biol. 2014; 59: 1400-1411.

52. Sancilio S, Gallorini M, Di Nisio C, Marsich E, Di Pietro R, Schweikl H, et al. Alginate/Hydroxyapatite-Based Nanocomposite Scaffolds for Bone Tissue Engineering Improve Dental Pulp Biomineralization and Differentiation. Stem Cells Int. 2018: Article ID 9643721.

53. Conde CM, Demarco FF, Casagrande L, Alcazar JC, Nör JE, Tarquinio SB. Influence of poly-L-lactic acid scaffold's pore size on the proliferation and differentiation of dental pulp stem cells. Braz Dent J. 2015; 26: 93-98.

54. Soares DG, Zhang Z, Mohamed F, Eyster TW, de Souza Costa CA, Ma PX. Simvastatin and nanofibrous poly(I-lactic acid) scaffolds to promote the odontogenic potential of dental pulp cells in an inflammatory environment. Acta Biomater. 2018; 68: 190-203.

55. Cavalcanti BN, Zeitlin BD, Nör JE. A Hydrogel Scaffold That Maintains Viability and Supports Differentiation of Dental Pulp Stem Cells. Dent Mater. 2013; 29: 97-102.

56. Feng X, Lu X, Huang D, Xing J, Feng G, Jin G, et al. 3D Porous Chitosan Scaffolds Suit Survival and Neural Differentiation of Dental Pulp Stem Cells. Cell Mol Neurobiol. 2014; 34: 859-870.

57. Zhang J, Lu X, Feng G, Gu Z, Sun Y, Bao G, et al. Chitosan scaffolds induce human dental pulp stem cells to neural differentiation: potential roles for spinal cord injury therapy. Cell Tissue Res. 2016; 366: 129-142.

58. Ullah I, Park JM, Kang YH, Byun JH, Kim DG, Kim JH, et al. Transplantation of Human Dental Pulp-Derived Stem Cells or Differentiated Neuronal Cells from Human Dental Pulp-Derived Stem Cells Identically Enhances Regeneration of the Injured Peripheral Nerve. Stem Cells Dev. 2017; 26: 1247-1257.

59. Zhang X, Zhou Y, Li H, Wang R, Yang D, Li B, et al. Intravenous administration of DPSCs and BDNF improves neurological performance in rats with focal cerebral ischemia. Int J Mol Med. 2018; 41: 3185-3194.

60. Pisciotta A, Riccio M, Carnevale G, Lu A, De Biasi S, Gibellini L, et al. Stem cells isolated from human dental pulp and amniotic fluid improve skeletal muscle histopathology in mdx/SCID mice. Stem Cell Res Ther. 2015; 6: 156.

61. Perczel-Kovach KE, Farkasdi S, Kallo K, Hegedus O, Keremi B, Cuisinier F, et al. Effect of stem cells of dental pulp origin on osseointegration of titanium implant in a novel rat vertebra model. Fogorv Sz. 2017; 110: 7-14.

62. Manimaran K, Sharma R, Sankaranarayanan S, Perumal SM. Regeneration of mandibular ameloblastoma defect with the help of autologous dental pulp stem cells and buccal pad of fat stromal vascular fraction. Ann Maxillofac Surg. 2016; 6: 97-100.

63. Li Y, Zhao S, Nan X, Wei H, Shi J, Li A, et al. Repair of human periodontal bone defects by autologous grafting stem cells derived from inflammatory dental pulp tissues. Stem Cell Res Ther. 2016; 7: 141.

64. Ferrarotti F, Romano F, Gamba MN, Quirico A, Giraudi M, Audagna M, et al. Human intrabony defect regeneration with micrografts containing dental pulp stem cells: A randomized controlled clinical trial. J Clin Periodontol. 2018; 45: 841-850.

65. Hernández-Monjaraz B, Santiago-Osorio E, Ledesma-Martínez E, AlcauterZavala A, Mendoza-Núñez VM. Retrieval of a periodontally compromised tooth by allogeneic grafting of mesenchymal stem cells from dental pulp: A case report. J Int Med Res. 2018; 46: 2983-2993. 